\title{
Birthing star forming clouds in the grand design
}

\author{
Yusuke Fujimoto $^{1}$,Elizabeth J. Tasker ${ }^{1}$, Greg L. Bryan ${ }^{2}$ and Asao \\ Habe $^{1}$ \\ ${ }^{1}$ Hokkaido University, Department of Physics, Faculty of Science, Sapporo 060-0810, Japan \\ ${ }^{2}$ Columbia University, Department of Astronomy, New York, NY 10025, USA
}

\begin{abstract}
We performed high resolution 3D hydrodynamical simulations of the barred galaxy M83 and investigated formation and evolution of the giant molecular clouds (GMCs) and star formation activity. We compared two simulations with and without stellar feedback. We found that the feedback disperses the cloud gas and causes clouds to inflow toward the galactic centre region due to hydrodynamical drag. The effect raises the star formation rate (SFR) and star formation efficiency (SFE) in the central bar region.
\end{abstract}

Keywords. hydrodynamics, methods: numerical, stars: formation, ISM: clouds, ISM: structure, galaxies: structure

\section{Introduction}

Understanding where and how gas is converted into stars within a galaxy affects every scale in astrophysics. In particular, it fills the gap between cosmological-scale galaxy formation and small-scale star formation in molecular cloud cores. What physical processes control the star formation in a galaxy is heavily debated.

Giant molecular clouds (GMC) are formed from the cold phase of the interstellar medium (ISM), where their densest pockets give birth to stars. The properties of the GMCs control the formation of these gravitationally unstable dense clumps and hence determine the rate of their star formation. Understanding the properties and distributions of the GMCs in a galaxy is therefore an important clue to understanding the star formation rate. Although there is a large difference in scale between GMCs and the galaxy, several theoretical works have succeeded in investigating the formation and evolution of GMCs while taking global galactic gas dynamics into account (e.g., Dobbs et al.2006; Tasker \& Tan 2009)

Galactic structures affect GMC distributions and properties. Fujimoto et al.(2014) performed hydrodynamical simulations of the barred galaxy M83 and found that distributions and properties of GMCs differ between galactic environments: bar, spiral arms, outer disc regions. Higher cloud density due to an elliptical gas motion caused by bar and spiral potential increases cloud interaction rates and changes GMC distributions and properties between the galactic regions.

Fujimoto et al.(2014) missed effects of stellar feedback on GMCs. Massive star larger than $8 M_{\odot}$ ejects huge energy $\left(\sim 10^{51} \mathrm{ergs}\right)$ into the ISM as supernova in the end of its life. In this proceeding, we show a barred galaxy simulation, which include star formation and stellar feedback.

\section{Numerical methods}

The isolated galaxy simulation presented in this proceeding was run using Enzo: a 3D adaptive mesh refinement (AMR) hydrodynamics code. We used a three-dimensional box of side $50 \mathrm{kpc}$ with a limiting resolution (smallest cell size) of about $1.5 \mathrm{pc}$. For the galaxy model, we used a static stellar potential from the nearby barred galaxy M83. Clouds are defined as coherent structures contained within contours at the threshold density of $n_{\text {gas }}=100 \mathrm{~cm}^{-3}$. Star formation and thermal stellar feedback are included. We performed two runs: no star formation or feedback run (NoSF) and thermal stellar feedback run (SNeHeat). 

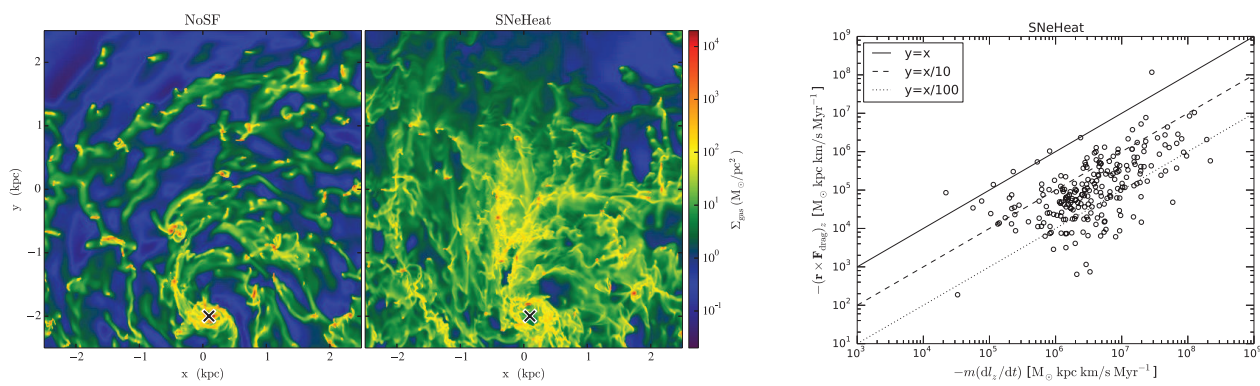

Figure 1. Left: zoom up images on the bar-end region of the galactic disc at $t=200 \mathrm{Myr}$ for NoSF and SNeHeat. Images show the gas surface density of the face-on disc. $x$ mark at the bottom of the image shows the galactic centre. The galactic disc rotates anticlockwise. Right: relation of $-\left(\mathbf{r} \times \mathbf{F}_{\mathrm{drag}}\right)_{z}$ versus $-m\left(\mathrm{~d} l_{z} / \mathrm{d} t\right)$ for each cloud. We exclude transient clouds, which are gravitationally unbound and have short lifetime.

\section{Results}

The left panels of Fig. 1 show zoom up images on the bar-end region of the galactic disc of NoSF and SNeHeat. The stellar feedback disperses part of the cloud gas, and the ISM density in inter-cloud region increases. The high inter-cloud density causes angular momentum loss of clouds due to hydrodynamical drag. The drag force, $\mathbf{F}_{\mathrm{drag}}$, is defined as $\mathbf{F}_{\mathrm{drag}}=\frac{1}{2} D \rho(\mathbf{v}-$ $\left.\mathbf{v}_{\mathrm{c}}\right)^{2} A \frac{\mathbf{v}-\mathbf{v}_{\mathrm{c}}}{\left|\mathbf{v}-\mathbf{v}_{c}\right|}$, where $D$ is a drag coefficient, $\rho$ is the density of the surrounding ISM of the cloud, $\mathbf{v}$ is the velocity of the ISM, $\mathbf{v}_{\mathrm{c}}$ is the cloud velocity, and $A$ is a cross sectional area of the cloud. The right panel of Fig. 1 shows the relation of $-\left(\mathbf{r} \times \mathbf{F}_{\mathrm{drag}}\right)_{z}$ versus $-m\left(\mathrm{~d} l_{z} / \mathrm{d} t\right)$ for each cloud in SNeHeat. $\mathbf{r}$ is a radial position of the cloud, $m$ is a cloud mass, and $l_{z}$ is a $z$ component of a specific angular momentum of cloud around the galactic centre. There is a clear relation between them. That means that normal clouds and especially massive clouds lose their angular momentum due to a torque from hydrodynamical drag. They inflow toward galactic centre, and then the total gas density in the central bar region rises. Moreover, the SFR and SFE increase compared with NoSF case.

\section{References}

Dobbs, C. L., Bonnell, I. A., \& Pringle, J. E. 2006, MNRAS, 371, 1663

Fujimoto, Y., Tasker, E. J., Wakayama, M., \& Habe, A. 2014, MNRAS, 439, 936

Tasker, E. J., \& Tan, J. C. 2009, ApJ, 700, 358 